\title{
Performance Analysis of a Low SAR Triangular Patch Textile Antenna for Health Monitoring Systems
}

\author{
A. Amsaveni, M. Bharathi
}

\begin{abstract}
In this article, a triangular patchtextile antenna has been proposed for health monitoring systems. The patch is fabricated using copper tape and substrate is made up of cotton. Two slots have been introduced near feedline to get larger the bandwidth.The size of the proposed antenna is $90 \times 80 \mathrm{~mm}^{2}$. The textile antenna is powered throughmicrostripfeedline structure. The antenna resonates at ISM band of $2.4 G H z$. The antenna provides a peak gainof $3.15 \mathrm{dBi}$ and radiation efficiency of $81.33 \%$ over the resonating frequency. The impacts on human head and handof electromagnetic radiation are evaluated. The electromagnetic radiation is evaluated usingSpecific absorption rate at different distances from human head and hand. The antenna parameters like radiation efficiency, radiation pattern, return loss and directivity have been evaluated. The antenna is designed and simulated using ANSYS High frequency structure simulator(HFSS) software and the fabricated prototype antenna is tested using network analyser.
\end{abstract}

Keywords-Triangular patch, Textile substrate,Specific Absorption Rate.

\section{INTRODUCTION}

Health care is one of the basic human rights and, as such, it should be considered asa benefit for all. Normally, the elderly peopleare often the affected category as Hospitals are not willingto spend their budget on services for elderly people. On the other side, the increase of life expectancy, together with the undeniable right of the elder to live ageing as a positive experience, motivates the need to find technological alternativesat low cost that could make health care provision more economical [1].

In this situation, telemedicine is regarded as one of the methodologies for significantly reducinghealth -related social expenses, while still offering essential assistance to the elderly people and ensuring a healthy quality of life. Wireless technology is a mainfactor for remote health monitoring. In fact, the combination of information technology and non-invasive wearable sensors can support the aged people to gettherequiredhelp,while living in their own homes, unlikeadmitted in costly hospitals.

The overallblock diagram of a health care monitoring system is shown in Fig.1. The following are thefourmainmodules:

(i) RF front end, which consists of a transceiver block for transmitting/receiving data to/from the data monitoring unit and an antenna.

Revised Manuscript Received on 14 August, 2019.

A.Amsaveni, Professor, Department of Electronics and Communication Engineering, Kumaraguru College ofTechnology, Coimbatore, Tamil Nadu, India. (Email:amsaveni.a.ece@kct.ac.in)

M.Bharathi, Professor, Department of Electronics and Communication Engineering, Kumaraguru College ofTechnology, Coimbatore, Tamil Nadu, India.
(ii)A Microcontroller unit for processing sensor information and sendingit to RF front end module.

(iii) Sensors

(iv) Power supply required for operating all the modules

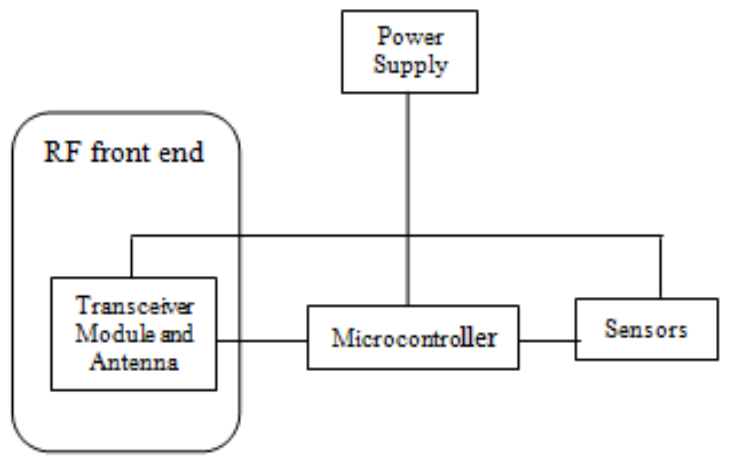

Fig.1.Overall Block diagram of a Health Monitoring System

Garment-integrated wearable products an important role in round-the-clock improvement of healthcare provisions. These wearable products not only alert and demandattention but also reducelabor and hospital resources. They also play a prominent role in preventative diseases, health disorders and heart or brain irregularities in people who are likely to be healthy[2].

All the four electronic blocks shown in Fig.1 should be incorporated into clothing or wearable items for efficient use of wearable wireless devices. It is therefore necessary to use non-conventional fabrication methods and materials in conjunction with customdesign approaches.

In the recent past, the wearable products has entered into the textile world. In general, Monitoring is a basic activity in endangered environments such as mountain climbing,diving,mining and military. However, medical monitoring is much neededfortransmittingmessages to hospital patients, home patients or perhaps outpatients. Allthese significant applications can therefore be accomplished with the help of wearable antennas which helps the wearer with such compact and durable products to stay in comfort zone [3].Wearable textile materials have been widely utilized for the development of microstrip antenna segment due to the latest miniaturization of wireless systems[4].Due to small weight, small size, and lowfabrication cost, small antennas such as microstrip antenna[5-6], RFID antenna, Fractal antenna, etchave been used in hand-held devices and other compact communication devices[7]. 
As the ground plane in microstrip antenna protects the body tissues and radiates perpendicularly to the planar structure, it is moreappropriateto use it in wearable applications [8].

By using a small dielectric substrate, increasing the substrate thickness, and using appropriate feeding methods, the bandwidth of wearable antennas can be improved[9]. The surface wave losses will be lower for small dielectric constant. Measuring the radiation absorbed by the human body as wearable antennas are closerthe human body is vital.Specific absorption rate (SAR) is used to measure the electromagnetic radiation absorbed by human body. SAR is a measure of the rate at which human body absorbs energy when it is subjected to radio frequency[10].

A triangular patch antenna for health monitoring applications is beingintended in this paper and its performance is being investigated. Normally, Textile antennas are widely used in wearable applications as they are cost effective, light weightand low profile structures.This wearable antenna can be fully integratedingarments and can be used to transmitor receive information from the sensor.

\section{METHODOLOGY}

The proposed patch antenna has a physical sizeof 80x90 $\mathrm{mm}^{2}$ and is supplied using microstripfeedlineby $50 \Omega$ transmission line. The triangular patch and ground plane are made up of copper tape. The substrate is made up of cotton materialwhose dielectric constant is 1.51 , thickness is 3.2 $\mathrm{mm}$ and loss tangent is 0.02 . Two slots are cut to provide a centre feed for the patch. Geometrical configuration of the proposed microstripantenna is depicted in Fig. 2.

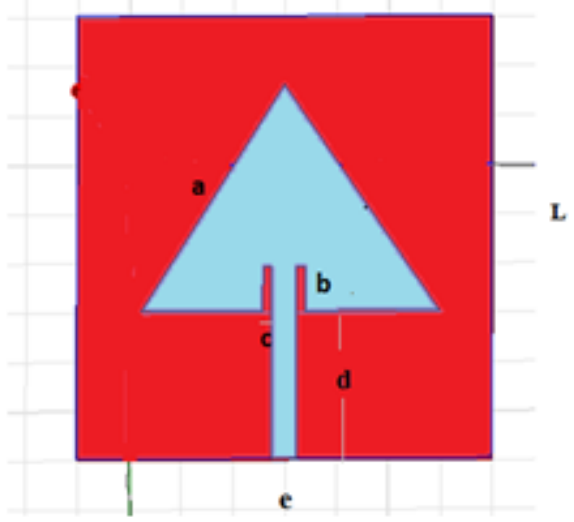

Fig.2. Structure of Proposed antenna

The resonant frequency of equilateral triangular patch is given as,

$f=2 c /(3 a \sqrt{\epsilon})------(2)$

where,

$\mathrm{a}$ is the vertex of triangle

$\mathrm{c}$ is the speed of light in free space

$\epsilon$ is the dielectric constant of the substrate

The triangular patch antenna has aground plane whose length (L)is $90 \mathrm{~mm}$ and width(W)is $80 \mathrm{~mm}$. Table 1 shows various dimensions of the proposed antenna. The triangle patch has a vertex (a) of $63 \mathrm{~mm}$. The length(b) of the slot is
$9 \mathrm{~mm}$ and width(c) is $1.5 \mathrm{~mm}$. These slots are made to provide a centre feed. The feed line has a dimension (e) of 4 $\mathrm{mm}$. The dimensions of the substrate is same as that of ground plane. The substrate is chosen with the thickness of $3.2 \mathrm{~mm}$

Table 1: Antenna Dimensions

\begin{tabular}{|l|l|}
\hline Dimensions & Values(mm) \\
\hline L & 90 \\
\hline W & 80 \\
\hline a & 63 \\
\hline b & 9 \\
\hline c & 1.5 \\
\hline d & 39 \\
\hline e & 4 \\
\hline
\end{tabular}

\section{RESULTS AND DISCUSSION}

\subsection{Simulation Results}

Using ANSYS HFSS 13.0 software, the triangular patch antenna is designed and simulated. The antenna parameters such as Return loss (S11), VSWR, Radiation Pattern, Gain and Efficiency are analysed.The simulated return loss S11of the antenna over frequency is depicted in Fig.3. It offers a return loss of approximately $-16 \mathrm{~dB}$ at resonant frequency.Lower the return loss, higher the radiated power

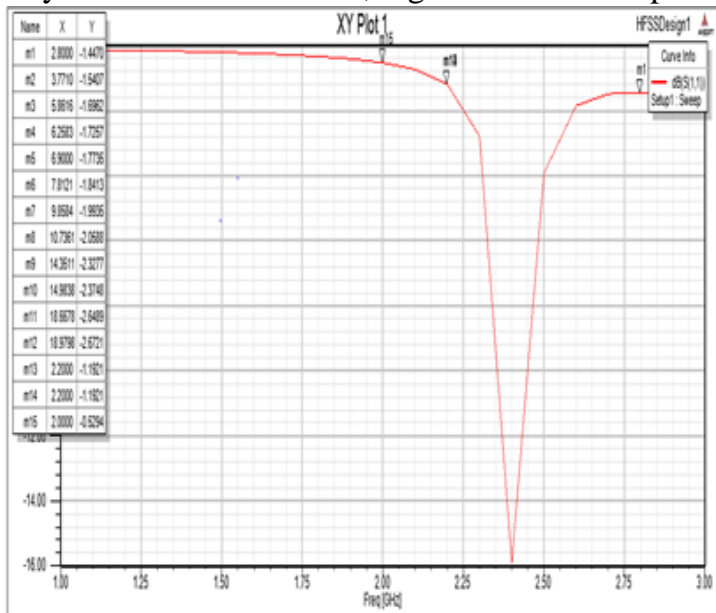

Fig.3.Return loss VsFrequency

Fig.4 shows the simulated antenna parameters of the proposed antenna at $2.4 \mathrm{GHz}$. The proposed antenna provides amaximumgain of $3.15 \mathrm{~dB}$, Front to back ratio of $50 \mathrm{~dB}$, and radiationefficiencyof $81.33 \%$. 


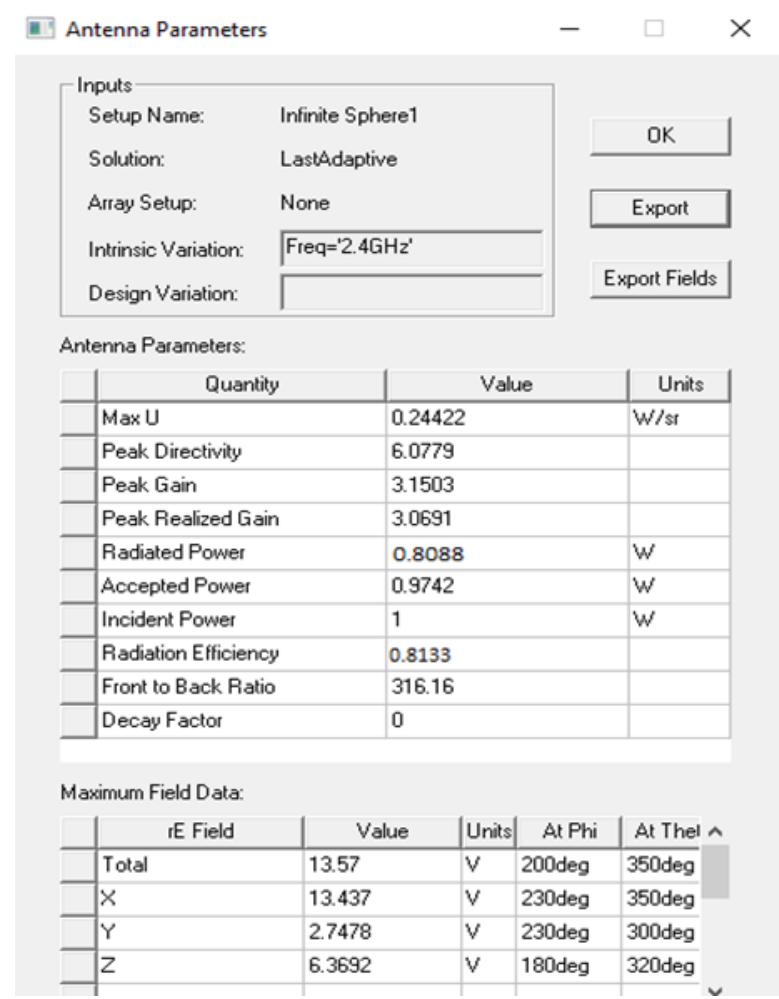

Fig.4 Simulated Antenna Parameters

The simulated 3D and 2D elevation patterns of the Textile antenna at resonant frequency are demonstrated in Fig.5 and Fig.6 respectively.

Here, the elevation pattern is noted, for phi $(\varphi)$ value of 90 degree and for all values of theta $\theta$. It is noted that the antenna exhibits symmetry uni-directional radiation pattern in elevation plane.
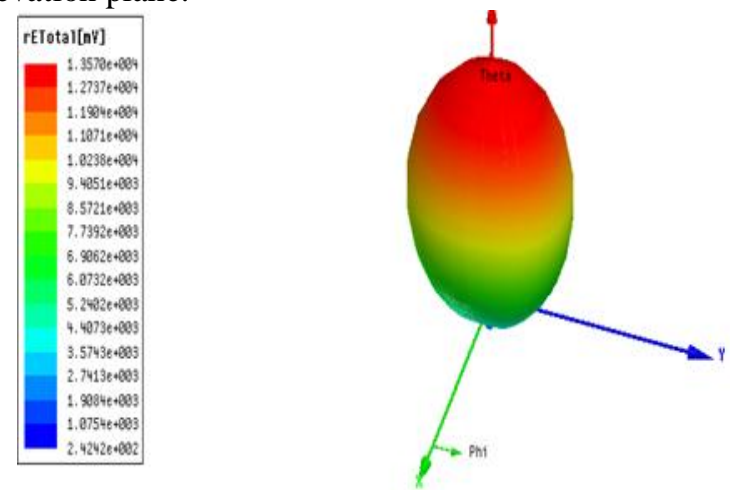

Fig 5. 3D Elevation Radiation Pattern
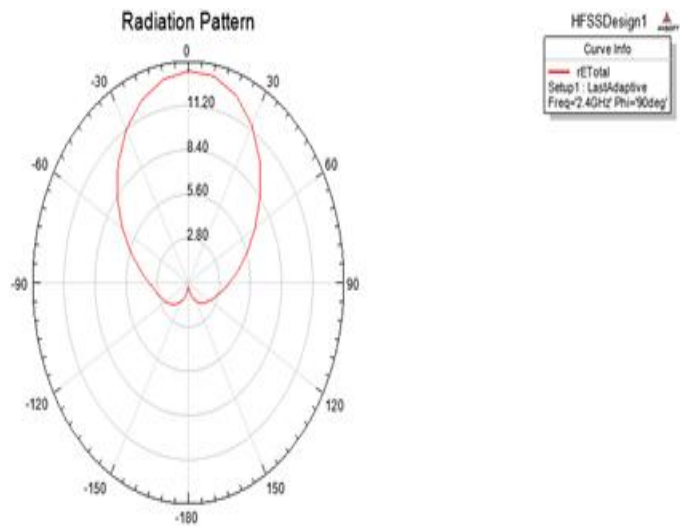

Fig.6 2D ElevationRadiation Pattern

\subsection{Measured Results}

The measured results of proposed antenna is discussed in this section. The designed antenna is fabricated and antenna parameters are measured using Microwave Vector Network Analyzer.

In the fabrication process of antenna, the substrate is made up of cotton material. Copper tapeis used to make the conducting patches and ground plane. Thecotton substrate is placed between triangular patch and ground plane.Fig. 7 gives the front and back view of the fabricated antenna.

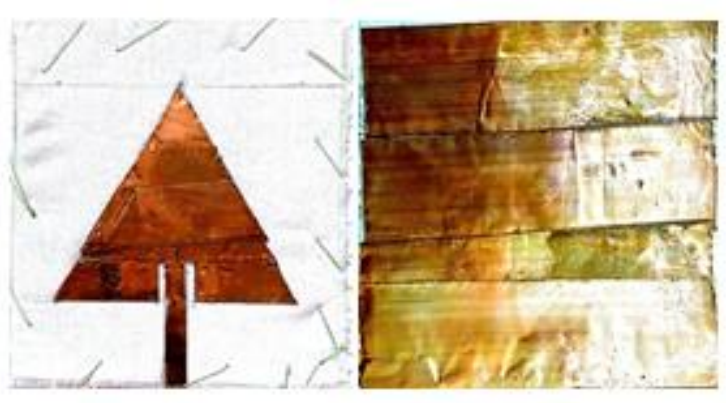

Fig.7 Front and back view of Prototype antenna

The measured results of proposed antenna is discussed in this section. The designed antenna is fabricated and antenna parameters are measured using Microwave Vector Network Analyzer.

Fig.8depicts the measuredReturn loss of the antenna.Thereturn loss of the antenna is approximately -11 $\mathrm{dB}$ at a frequency of $2.4 \mathrm{GHz}$.Fig. 9 shows the measured VSWR of the antenna. It provides a VSWR of 1.9.

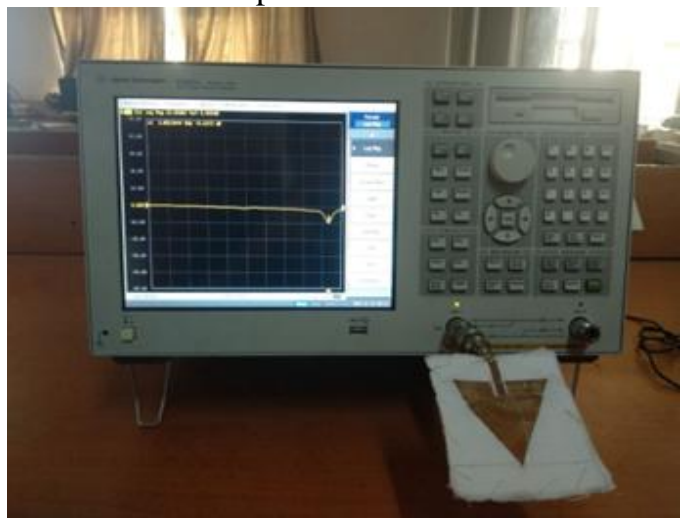

Fig.8Return loss plot of the Prototype Antenna

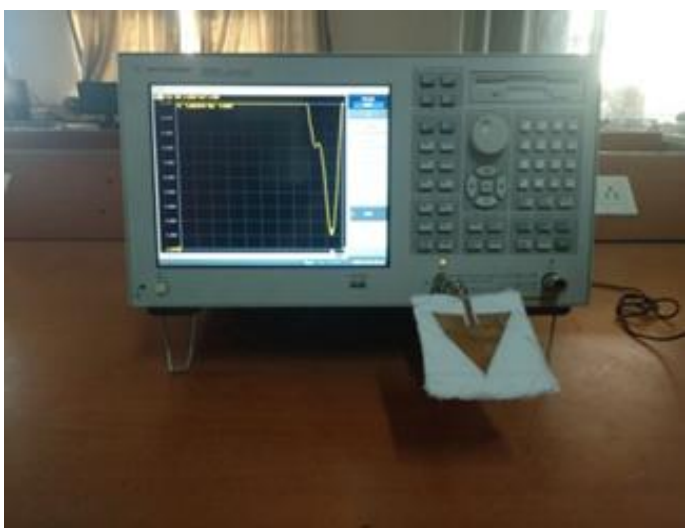

Fig.9 VSWR of the Prototype Antenna 


\section{PERFORMANCE ANALYSIS OF A LOW SAR TRIANGULAR PATCH TEXTILE ANTENNA FOR HEALTH MONITORING SYSTEMS}

\subsection{SAR Calculationusing HFSS}

ANSYS HFSS is used for the measurement of Specific Absorption rate (SAR).SAR is a measure of the rate of energy absorption by thehuman body when subjected to RF. It is described as the power that is absorbed by tissuemass and has unit of watts per kilogram (W/kg) [11]. It is possible to calculate SAR for electromagnetic energy from the electric field within the tissue as:

$$
\mathrm{SAR}=\frac{1}{V} \int \frac{\sigma(r)|E(r)|^{2} 2}{\rho(r)} d r
$$

$\sigma$ is the sample electrical conductivity, E is the RMS electric field, $\square$ is the sample density, $\mathrm{V}$ is the volume of the sample. The peak SAR values (spatial-peak SAR [IEEE1529]) are averaged over $1 \mathrm{~g}$ and $10 \mathrm{~g}$ of human tissues by setting excitation equivalent to 0.6 Watts. Usually, SAR is averaged either over the entire body, or over a small sample volume.The permissible or preferred SAR values are $2 \mathrm{~W} / \mathrm{kg}$ per $10 \mathrm{~g}$ and $1.6 \mathrm{~W} / \mathrm{kg}$ per $1 \mathrm{~g}$ of body tissue. [12]. The SAR value also relies on the substrate'sdielectric properties. Each substrate depositsdistinct amounts of radiation from the antenna to the human body.

A numericalhand and head phantom modelsof Specific Anthropomorphic Mannequin (SAM) [13] are used in this research work. SAR calculator is provided by the HFSS software. It enables the local and the average SAR to be known in distinct volumes of the antenna design. Thus, a volume with distinct layers can be created that represents some portion of the human body. The most significant thing to do this is to understand the features of distinct body layers that represent the distinct human tissues of that portion of the body.

Fig.10 and Fig.11 show the SAR analysis of a proposed triangular patch antenna for both hand and head respectively. The maximum and minimum SAR values for hand are $0.96 \mathrm{~W} / \mathrm{Kg}$ and $0.08 \mathrm{~W} / \mathrm{Kg}$ over $1 \mathrm{~g}$ of tissue respectively. The maximum and minimum SAR values for head are $0.51 \mathrm{~W} / \mathrm{Kg}$ and $0.08 \mathrm{~W} / \mathrm{Kg}$ over $1 \mathrm{~g}$ of tissue respectively. Table 2 summarizes the SAR values.

Table 2. SAR Values for Textile Antenna

\begin{tabular}{|c|c|c|}
\hline \multirow{2}{*}{} & \multicolumn{2}{|c|}{ SAR(W/kg)-1g of body tissue } \\
\cline { 2 - 3 } & $1 \mathrm{~cm}$ from Hand & $1.5 \mathrm{~cm}$ from Head \\
\hline Min.SAR & 0.08 & 0.08 \\
\hline Max.SAR & 0.96 & 0.51 \\
\hline
\end{tabular}

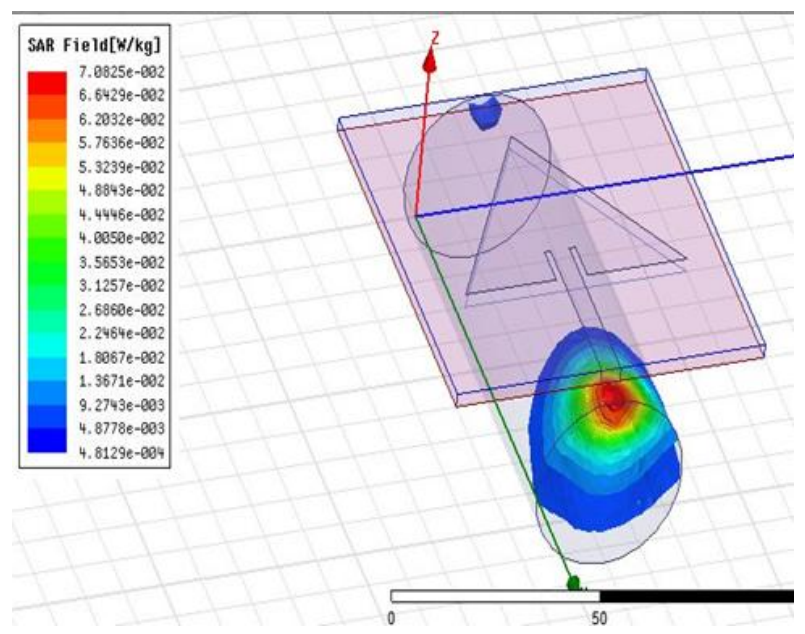

Fig.10SAR analysis for hand

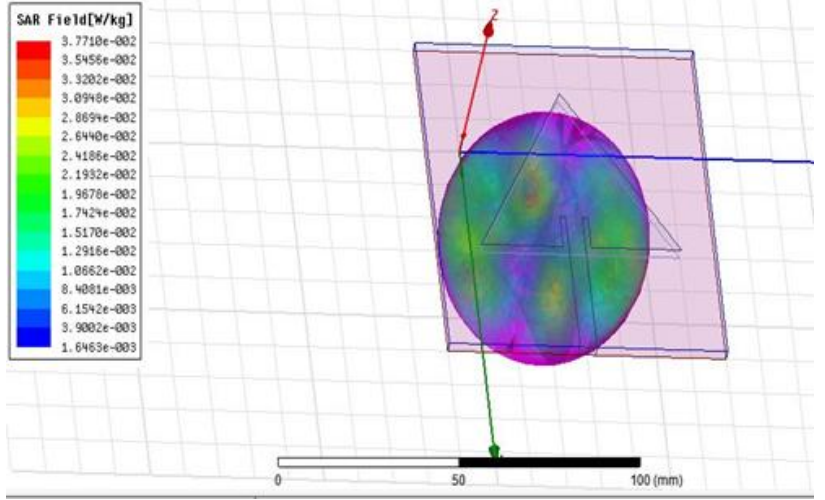

Fig.11 SAR analysis for head

\section{IV.CONCLUSION}

The proposed article presents a triangular patch textile antenna for health monitoring applications. The patch is fabricated using copper tape and substrate is made up of cotton. The physical size of the proposed antenna is $90 \times 80$ $\mathrm{mm}^{2}$ and is fed by micro strip line feed structure. The proposed antenna structure resonates at $2.4 \mathrm{GHz}$. The radiation efficiency is $81.33 \%$ and peak antenna gain is 3.15 dBiat the resonantfrequency. Hence the proposed antenna is appropriate for wireless health monitoring applications. The simulated and measured resultsare used to validate the fabricated antenna; the measured results are better than the simulation results. The proposed workalsoanalysed the impacts of electromagnetic radiation on the human head and hand in terms of SAR. SAR ismeasured at distinct distances from antenna to the human head and hand. The maximum and minimum SAR values for hand are $0.96 \mathrm{~W} / \mathrm{Kg}$ and $0.08 \mathrm{~W} / \mathrm{Kg}$ over $1 \mathrm{~g}$ of tissue respectively. The maximum and minimum SAR values for head are $0.51 \mathrm{~W} / \mathrm{Kg}$ and $0.08 \mathrm{~W} / \mathrm{Kg}$ over $1 \mathrm{~g}$ of tissue respectively. Because of the low SAR values the proposed antenna can be used for communication and biomedical applications.

\section{REFERENCES}

1. Bonato P. Wearable sensors and systems: From enabling technology to clinical applications. IEEE Engineering in Medical and Biology Magazine. 29:25-36,2010

2. Bariya, M., Nyein, H. \&Javey, A. Wearable sweat sensors, Nature Electronics. 1, 160-171,2018.

3. Mukhopadhyay, S.C. Wearable Sensors for Human Activity Monitoring: A Review. Sensors Journal, IEEE. 15. 1321-1330, 2015.

4. Odame, Kofi \& Du, Dingkun. Towards a smart sensor interface for wearable cough monitoring. Proceedings of IEEE Global Conference on Signal and Information Processing,654-657,2013.

5. A. Amsaveni,M. Bharathi, J. N. Swaminathan, Design and performance analysis of low SAR hexagonal slot antenna using cotton substrate, Microsystem Technologies 25:2273-2278,2019

6. A. Amsaveni, Antennas and Wave Propagation. Anuradha Publications, Chennai, 2017.

7. A. Amsaveni,K. Anusha,A circularly polarized triangular slot reconfigurable antenna for wireless applications,International Journal of Pure and Applied Mathematics,116(11), 81-89,2017.

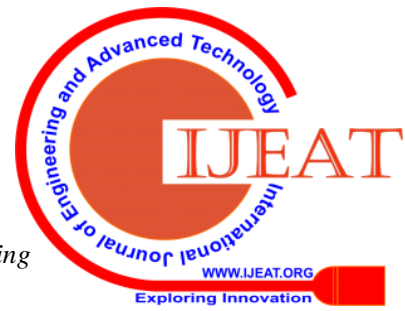


8. Varshney, S, Malhotra.M, Sharma S\& Aggarwal, P. (2018). Specific absorption rate (SAR) value of mobile phones: An awareness study among mobile users. Indian Journal of Community Health. 30. 156-162,2018.

9. A. RegiSaral, V. Lavanya, A. Amsaveni, A Triangular Patch Antenna for Wireless Applications",Intl.Journal of Advanced Research in Electronics and Communication Engineering, 7(3), ISSN: 2278 - 909X,2018.

10. A K M FazlulHoque, Md. Sazzad Hossain, ASattarMollah, Md. Akramuzzaman. A Study on Specific Absorption Rate (SAR) Due to Non-Ionizing Radiation from Wireless/Telecommunication in Bangladesh. American Journal of Physics and Applications.1(3):104-110,2013.

11. Hengyi Zhou, Arpan Pal, Amit Mehta, Low SAR Capacitively Coupled Compact Multiband Antenna for SmartphonesIEEE Loughborough Antennas \& Propagation Conference (LAPC), 978-1-4799-8943$0 / 15,2015$.

12. Mohammad Rashed Iqbal Faruque, Mdlkbal Hossain, Mohammad Tariqul, Low Specific Absorption Rate Microstrip Patch Antenna for Cellular Phone Applications, IET Microwave Antennas and Propagation, 9(14),1540-1,2015.

13. IkbalHossainaMd, Iqbal Faruquea M R, TariqulIslamb $\mathrm{M}$, Investigation of Hand Impact onPIFA performances and SAR in Human Head, Journal of Applied Research and Technology, 13,447-453,2015. 